\title{
Experimental study of hypersonic boundary layer flow control based on isolated roughness
}

\author{
Shao Zhongjie, Chen Xing, Wang Dan, Yuan Xiangjiang \\ China Academy of Aerospace Aerodynamics \\ Beijing, China \\ Shaozhongjie229@163.com
}

\begin{abstract}
A boundary layer flow control device based on isolated roughness elements is proposed, and experimental verification tests are carried out in FD-20 wind tunnel of CAAA. The experimental Mach number is 6, and Reynolds number is $1 \mathrm{E}+7 / \mathrm{m}$. The heat flux sensors are used to capture the boundary layer transition position and heat flux distribution. When the height is appropriate, a stable streamwise vortex structure can been formed downstream of the roughness element. This steady vortex can change the original boundary layer structure. The transition can be postponed and the heat flux in turbulent boundary layer can be reduced. In the paper, roughness can effectively delay transition position by $30 \mathrm{~mm}$ and reduce heat flux in turbulent region by about $4-13 \%$.
\end{abstract}

\section{Introduction}

Laminar-turbulent transition in boundary layer is important for the design of hypersonic vehicles. The heat transfer of hypersonic flight vehicles depend on the flow state of the boundary layer. While the boundary layer is turbulent, heat transfer on vehicles increase twice at least ${ }^{[1]}$. The impacts make transition important in the process of designing hypersonic vehicle, especially designing of thermal protection system (TPS).

For essentially two-dimensional (2-D) hypersonic flows, the initial phase of laminar-turbulent transition is associated with amplification of the second mode ${ }^{[2,3]}$. This mode belongs to the family of trapped acoustic waves propagating in the wave-guide, which is formed within the boundary layer. Fedorov and Malmuth ${ }^{[4,5]}$ assumed that a thin porous coating may suppress the second and higher acoustic modes and, at the same time, may not trip the boundary layer due to roughness of the porous surface. The stability analyses showed that such an ultrasonically absorptive coating (UAC) reduces the secondmode growth rate dramatically. Maslov et $\mathrm{al}^{[6]}$ also found that a passive porous coating of random structure (felt metal) significantly delays transition on a sharp cone at zero angle of attack in the Mach=12 wind tunnel.

However, there is little study which was performed to reduce heat flux in hypersonic turbulence boundary layer. In this paper, experimental study of hypersonic boundary layer flow control based on isolated roughness were carried out at freestream Mach numbers 6 in FD-20 wind tunnel of CAAA, to reduce aeroheating in turbulence boundary layer and delay the transition.

\section{Experimental facility, model and conditions}

\section{1. Experimental facility}

FD-20 is a hypersonic piston gun wind tunnel at China Academy of Aerospace Aerodynamic. The facility is a piston shot gun tunnel with a nozzle exit of $380 \mathrm{~mm}$ in diameter. Free stream Mach number is set with different Laval contour nozzles from 5 to 15. All the transition experiments are made at Mach 6. The typical stable flow period varies from 25 to 60 milliseconds. In order to reduce the background noise disturbance, low total pressure state is used in wind tunnel operation, at which the unit Reynolds number is about $1.0 \mathrm{E}+7 /$ meter. 


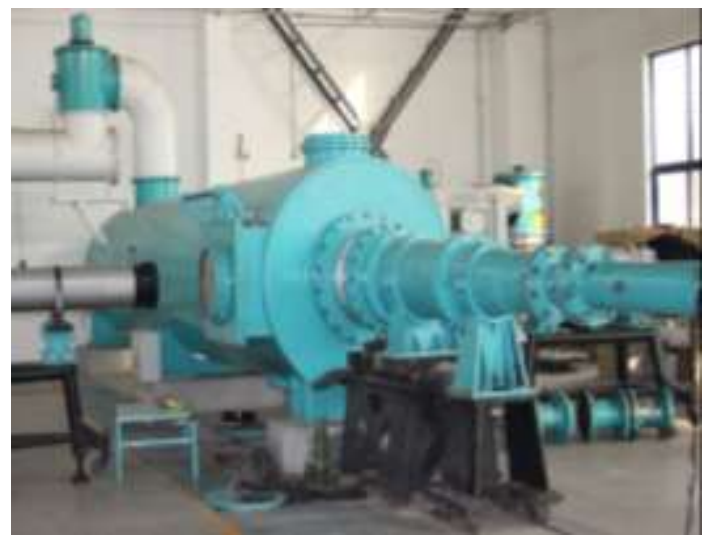

Fig. 1: FD-20 piston gun wind tunnel.

\section{2 Experimental model}

The experimental model is shown in figure 2. It is a stainless-steel flat plate with a length of $500 \mathrm{~mm}$ and a width of $320 \mathrm{~mm}$. Four groups of roughness were installed on the plate, whose location is $x=100 \mathrm{~mm} 、 150 \mathrm{~mm} 、 320 \mathrm{~mm}$ and $370 \mathrm{~mm}$, and the height of roughness is $0.3 \mathrm{~mm} 、 0.3 \mathrm{~mm} 、 1.0 \mathrm{~mm}$ and $1.2 \mathrm{~mm}$. The space of roughness is $12 \mathrm{~mm}$. Roughness elements can be arranged in two ways: "cross" and "along". "cross" means that two adjacent groups of roughness elements are interlaced, and "along" means that two adjacent groups of roughness elements are on the same streamline. The heat flux sensors are installed along streamwise direction, and the installed locations of sensors are divided into seven lines (L1 L7), which were shown in table 1.

Table 1: The location of measurement line.

\begin{tabular}{|c|c|}
\hline $\begin{array}{c}\text { The name of measurement } \\
\text { line }\end{array}$ & $\begin{array}{c}\text { The location of } \\
\text { measurement line }\end{array}$ \\
\hline L1 \& L5 & $\begin{array}{c}\text { placed between the two } \\
\text { roughness }\end{array}$ \\
\hline L2 \& L6 & $\begin{array}{c}\text { along the outer edge of } \\
\text { roughness }\end{array}$ \\
\hline L3 \& L7 & along the center of roughness \\
\hline L4 & Center line of plate \\
\hline
\end{tabular}
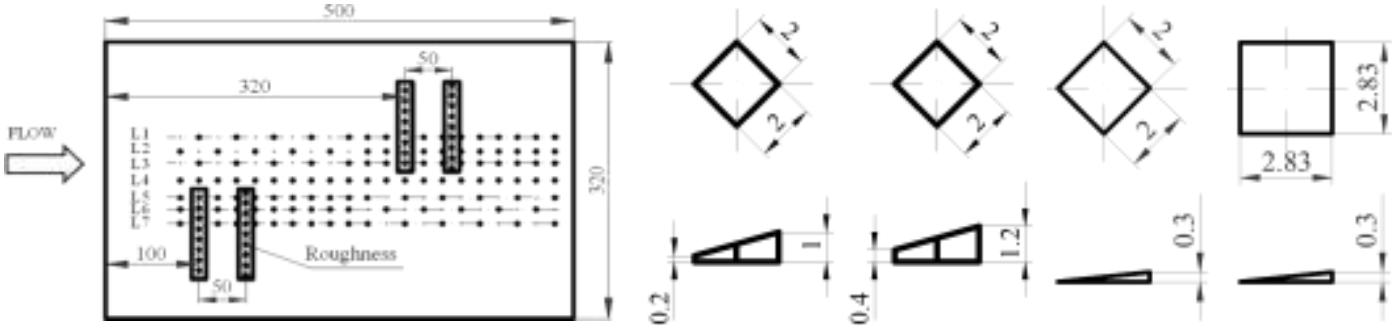

$-2.83$

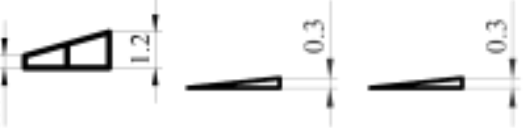

Fig. 2: Sketch of shape and size of roughnesses. 


\section{3. Experimental conditions}

The experiments were carried out at Mach numbers 6 . Reynolds number is $1 \mathrm{E}+7 / \mathrm{m}$. The total pressure and temperature are $3 \mathrm{MPa}$ and $930 \mathrm{~K}$.

\section{Experimental results}

The experiments are divided into two parts: two groups of roughness elements $\mathrm{x}=100 \mathrm{~mm}$ and $150 \mathrm{~mm}$ are used to delay boundary layer transition; two other groups are used to reduce heat flux in turbulent boundary layer.

Figure 3 shows the experimental results of reducing heat flux in turbulent boundary layer by the roughness element device. The dotted red line indicates the mounting position of the roughness element devices. The heat flux in the turbulent region of Line L1 and Line L3 is significantly lower than the value of the undisturbed plate. In the case "cross", the values of L1 and L3 are reduced by $13.02 \%$ and $11.30 \%$ respectively; In the case "along", the values are reduced by $8.61 \%$ and $11.13 \%$ (see Table.2). The heat flux of L2 also decrease slightly about 4-5\%.

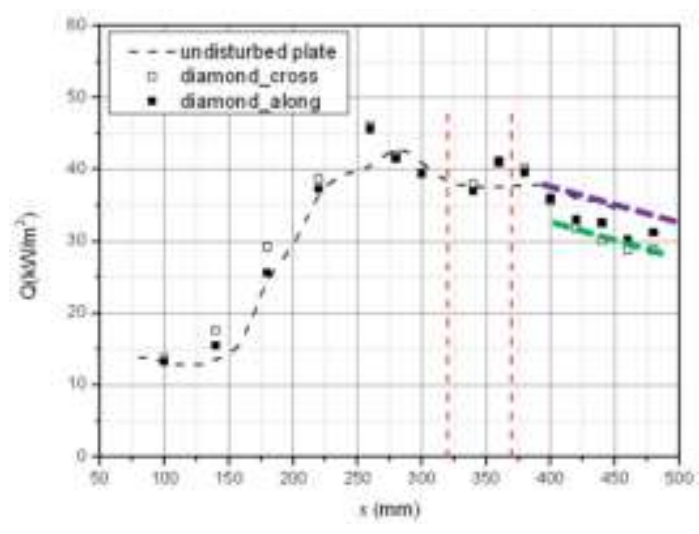

(a) L1 (between the two roughness)

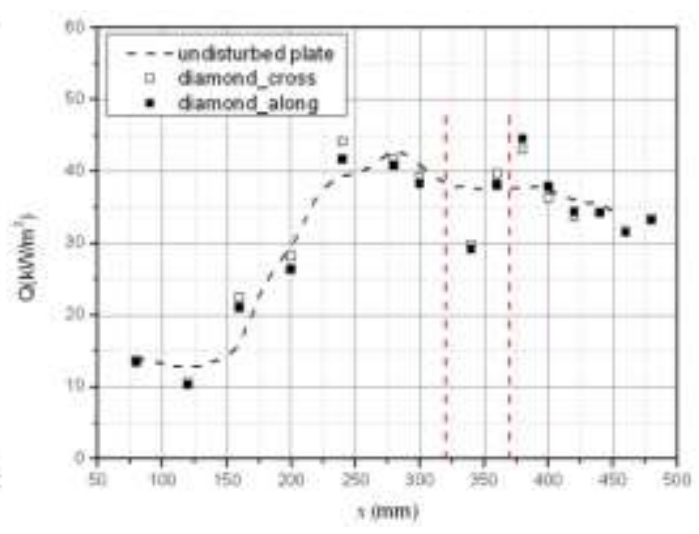

(b) L2 (along outer edge of roughness)

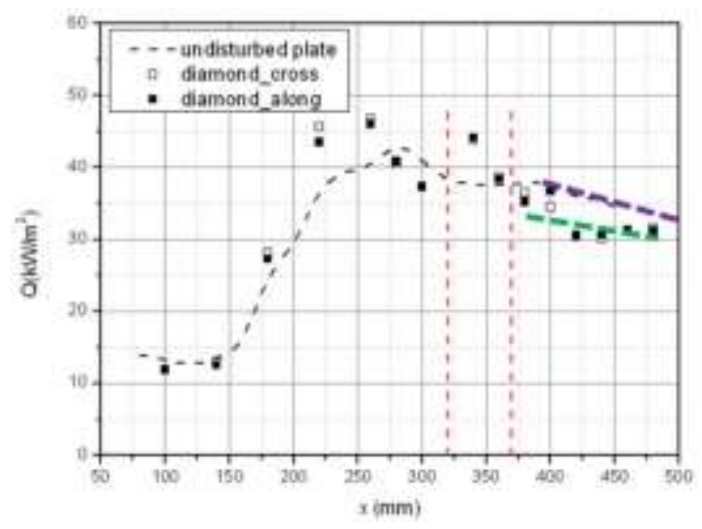

(c) L3 (along the center of roughness)

Fig. 3: Experimental results of reducing heat flux. 
Table 2: Comparison of heat flux values with or without roughness element devices.

\begin{tabular}{|c|c|c|c|c|c|c|c|}
\hline & & \multicolumn{3}{|c|}{ Diamond_cross } & \multicolumn{3}{|c|}{ Diamond_along } \\
\hline $\mathrm{x}(\mathrm{mm})$ & $\begin{array}{l}\text { Undisturb } \\
\text { ed } \\
\text { plate }\end{array}$ & L1 & L2 & L3 & L1 & L2 & L3 \\
\hline 400 & 37.78 & 35.55 & $1^{36.3}$ & 34.52 & $6^{35.9}$ & $3^{37.9}$ & 36.69 \\
\hline 420 & 35.65 & 31.89 & $1^{33.6}$ & 30.68 & $7^{33.0}$ & $4^{34.3}$ & 30.48 \\
\hline 440 & 35.62 & 30.14 & $8^{34.2}$ & 30.22 & $4^{32.5}$ & $6^{34.2}$ & 30.65 \\
\hline 460 & 33.64 & 28.78 & $8^{31.7}$ & 31.23 & $3^{30.2}$ & $8^{31.4}$ & 31.28 \\
\hline 480 & - & 28.78 & $3^{33.4}$ & 31.56 & $1^{31.2}$ & $0^{33.2}$ & 31.19 \\
\hline $\begin{array}{l}\text { Mean } \\
\text { value }\end{array}$ & 35.67 & 31.03 & $8^{33.8}$ & 31.64 & $0^{32.6}$ & $4^{34.2}$ & 32.06 \\
\hline $\begin{array}{l}\text { Reduci } \\
\text { ng heat flux }\end{array}$ & $0 \%$ & $\%^{13.02}$ & $\%^{5.01}$ & $\%^{11.30}$ & $\%^{8.61}$ & $\%^{4.00}$ & $\%^{11.13}$ \\
\hline
\end{tabular}

Figure 4 shows the experimental results of delayed boundary layer transition by the roughness element device. The transition position of Line L5 and Line L7 obviously moves backward. Transition position is delayed about 30mm. However, the position of transition on Line L6 is slightly changed, and transition position of case "diamond+square_long" moves forward.

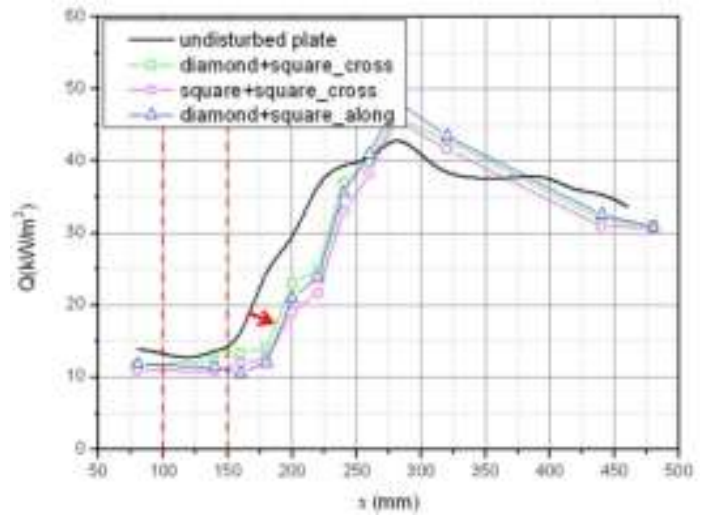

(a) L5 (between the two roughness)

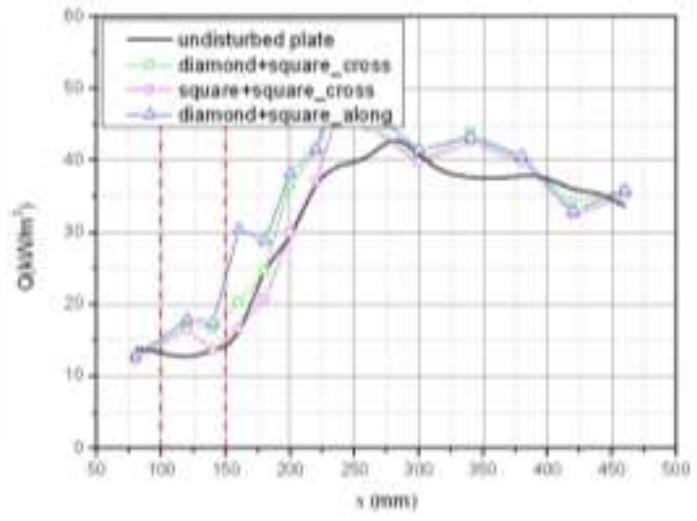

(b) L6 (along outer edge of roughness) 


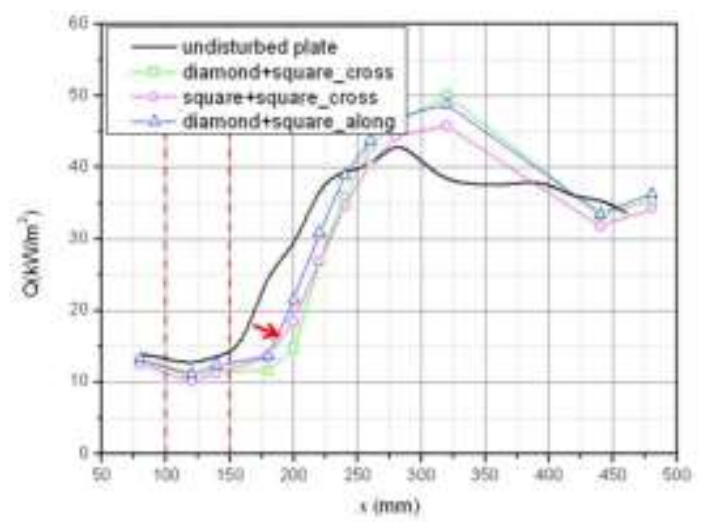

(c) L7 (along the center of roughness)

Fig. 4: Experimental results of delaying transition.

The results of Directory Numerical Simulation (DNS) (Figure.5) show that, a stable streamwise structure can be formed downstream of a rough element with a specific height of shape. When the roughness element appears in the laminar boundary layer, the flow structure in wake of roughness and the unstable structure formed by the disturbances of the incoming flow interact with each other, changing the original transition process and thereby delaying transition. When the roughness appears in the turbulent boundary layer, the streamwise structure induced by roughness will interact with the turbulent structure in the boundary layer, thereby inhibiting the turbulence and achieving the heat flux reduction.

In general, the wake of roughness element is a stable structure of streamwise vortex, and the flow control effect is obvious in the region swept by the streamwise vortex, but the effect is not obvious where there is no streamwise vortex. This also explains that the downstream of the center line of roughness has the best control effect, while the downstream of the outer edge of the roughness has the worst control effect.

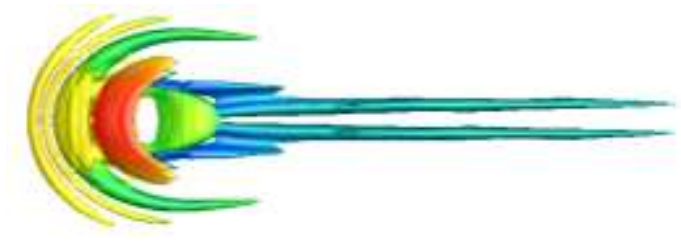

Fig. 5: The streamwise vortex induced by roughness element.

\section{Conclusion}

In this paper, a boundary layer flow control device based on isolated roughness elements is proposed, and experimental verification tests are carried out in FD-20 wind tunnel. The experimental Mach number is 6 , and Reynolds number is $1 \mathrm{E}+7 / \mathrm{m}$. The heat flux sensors are used to capture the boundary layer transition position and heat flux distribution.

When the height is appropriate, a stable streamwise vortex structure can been formed downstream of the roughness element. This steady vortex can change the original boundary layer structure. The transition can be postponed and the heat flux in turbulent boundary layer can be reduced. In the paper, roughness can effectively delay transition position by $30 \mathrm{~mm}$ and reduce heat flux in turbulent region by about $4-13 \%$.

In fact, these are preliminary experimental results. The flow control mechanism of the isolated roughness elements mentioned in this paper is only a guess of authors. This speculation, though partially validated by numerical calculations, is not sufficient. Further research is needed. 


\section{References}

[1] H. H. Hamilton II, D. R. Millman, R. B. Greendyke, "Finite difference solution for laminar or turbulent boundary layer flow over axisymmetric bodies with ideal gas, CF4, or equilibrium air chemistry," Technical Paper 3271, NASA, December 1992.

[2] L. M. Mack, "Boundary-layer linear stability theory," Rep. AGARD 709, Jet Propul. Lab., Pasadena, CA., 1984.

[3] L. M. Mack, "Linear Stability Theory and the problem of supersonic boundary layer transition," AIAA Journal, vol. 13, no. 3, pp. 278-289, March 1975.

[4] N. D. Malmuth, A. V. Fedorov, V. Shalaev, J. Cole, \& A. Khokhlov, "Problems in high speed flow prediction relevant to control," AIAA Paper 98-2695.

[5] A. V. Fedorov, N. D. Malmuth, "Stabilization of hypersonic boundary layers by porous coatings," AIAA J., vol. 39, pp. 605-610, 2001.

[6] A. Maslov, A. Shiplyuk, A. Sidorenko A, "Hypersonic Laminar Flow Control Using a Porous Coating of Random Microstructure," AIAA 2006-1112. 